\title{
Urinary incontinence and mortality among older adults residing in care homes
}

This is the peer reviewed version of the following article: Damián J, Pastor-Barriuso R, García López FJ, de Pedro-Cuesta. Urinary incontinence and mortality among older adults residing in care homes. J Adv Nurs. 2017 Mar;73(3):688-699, which has been published in final form at https://doi.org/10.1111/jan.13170. This article may be used for non-commercial purposes in accordance with Wiley Terms and Conditions for Use of Self-Archived Versions

Javier DAMIÁN; Roberto PASTOR-BARRIUSO; Fernando GARCÍA-LÓPEZ; Jesús DE PEDRO-CUESTA.

Authors' affiliations: National Centre for Epidemiology, Carlos III Institute of Health, Madrid, Spain (All authors). Consortium for Biomedical Research in Neurodegenerative Diseases (Centro de Investigación Biomédica en Red sobre Enfermedades Neurodegenerativas - CIBERNED), Madrid, Spain (Javier DAMIÁN, Jesús DE PEDRO-CUESTA, Fernando GARCÍA-LÓPEZ). Consortium for Biomedical Research in Epidemiology and Public Health (CIBER en Epidemiología y Salud Pública - CIBERESP), Madrid, Spain (Roberto PASTOR-BARRIUSO).

Correspondence author: Javier Damián. Centro Nacional de Epidemiología. Instituto de Salud Carlos III. Av/ Monforte de Lemos 5; 28029-Madrid, Spain. Telephone number: +34 91822 2230. Fax: +34 91822 2908; e-mail: jdamian@isciii.es.

Funding: This work was supported by grant PI15CIII/00037 from the "Carlos III" Institute of Health. Conflict of interest. No conflict of interest has been declared by the authors.

Running head: Incontinence and mortality.

"This is the peer reviewed version of the following article: [J Adv Nurs. 2017; 73 (3): 688-699.], which has been published in final form at [https://doi.org/10.1111/jan.13170]. This article may be used for noncommercial purposes in accordance with Wiley Terms and Conditions for Use of Self-Archived Versions." 


\section{ABSTRACT}

Aim. To assess the association between baseline urinary incontinence and long-term, all-cause mortality.

Background. Urinary incontinence is a common disorder among older institutionalised adults, with important consequences for morbidity and quality of life. Moreover, while it is a consistent mortality marker, the extent to which this association might be causal remains controversial.

Design. A cohort study.

Methods. We conducted a mortality follow-up study on a cohort of 675 nursing-home residents in the city of Madrid (Spain), from their 1998-1999 baseline interviews to September 2013. Study subjects or their caregivers were asked whether the resident had experienced any involuntary leakage of urine in the preceding 14 days, with subjects being subsequently defined as continent, mildly incontinent or severely incontinent. Hazard ratios for all-cause mortality were estimated using Cox proportional hazards models.

Results. After a 4061 person-year follow-up (median/maximum of 4.6/15.2 years), 576 participants had died. In fully-adjusted models, urinary incontinence was associated with a twenty-four percent increased risk of all-cause mortality. There was a graded relationship across severity levels, with hazard ratios seven percent higher for mild and forty-four percent higher for severe incontinence as compared with the continent group. The adjusted mortality fraction attributable to urinary incontinence was eleven percent.

Conclusion. It would appear that urinary incontinence is not only a marker but also a real determinant of survival in the institutionalized population. This finding, which seems plausible in a population of frail older adults, warrants further research into mechanisms that could help to elucidate this hypothesis. 
Keywords: urinary incontinence, mortality, nursing homes, long-term care, aged, homes for the aged, nursing, epidemiology, cohort study, proportional hazards models 
SUMMARY STATEMENT

\section{Why is this research or review needed?}

- Urinary incontinence is a prevalent condition in nursing homes with relevant consequences for health, quality of life and cost.

- While urinary incontinence has long been established as a mortality marker, due to its role in geriatric syndromes and association with frailty, a direct causal role nonetheless remains doubtful.

\section{What are the key findings?}

- Our study shows a consistent, graded association with mortality in a representative population of nursing-home residents, after adjustment for a relevant set of confounding factors.

- Furthermore, it furnishes novel estimates of how the effect, measured by hazard ratios, is not constant but changes with the passage of time.

\section{How should the findings be used to influence policy/practice/research/education?}

- Preventing or improving urinary incontinence through adequate care and attention may increase survival rates among nursing-home residents, an additional benefit to the indisputable improvements in quality of life achieved by successful interventions addressing this highly prevalent condition.

- Further research is needed to understand the underlying mechanisms. Increased risk for falls and infections and decreased mobility and social participation could be potential mechanisms to study. Cluster randomised intervention trials may be the appropriate design to tackle those research objectives. Research is also needed into the specific role of nursing to better manage urinary incontinence in nursing homes. 


\section{INTRODUCTION}

Urinary incontinence is defined as the complaint of any involuntary loss of urine (Abrams et al. 2010), affecting millions of people worldwide, with a substantial economic burden to patients and society (Milsom et al. 2014). It is a prevalent condition in nursing homes, with half of all residents affected to a significant degree (Jones et al. 2009). A recent systematic review reported prevalence figures of between $43-77 \%$ in the institutionalised population (Offermans et al. 2009).

Urinary incontinence is considered one of several interrelated geriatric syndromes and its consequences may trigger adverse outcomes for frail older people (Johnson et al. 2000, HolroydLeduc et al. 2004, Berardelli et al. 2013). The principal defining features of frailty are reduced physiological reserve and increased vulnerability to adverse outcomes; it is highly prevalent in nursing home populations (Kojima 2015).

\section{Background}

The association between urinary incontinence and mortality has given rise to disparate conclusions in the literature. In a large-scale study of an older community-dwelling population in California, Thom et al. (1997) reported an association with clear increases in mortality among men and women, of $20 \%$ and $10 \%$, respectively. However, their study drew no distinction between urinary and faecal incontinence (they estimated that $6 \%$ of subjects had isolated faecal incontinence). While Herzog et al. (1994) failed to find an association, their study population had a generally better health status than did ours, not only because of their younger age profile, but also because they adjusted for subjects' self-assessment of health and self-assessment necessarily limits the sample to those who are able to provide a valid assessment. Tilvis et al. (1995) observed an association that disappeared once the estimate had been adjusted for dementia and 
Johnson et al. (2000) observed no adjusted association. In a 10-year follow up study, Nuotio et al. (2002) found urge incontinence associated with mortality in a population of older Finnish men, which included a small fraction of institutionalised individuals; this association was not adjusted for dementia or cognitive level and was clearer in men. Holroyd-Leduc et al. (2004) did not find an adjusted association.

Roughly midway between a community-dwelling and nursing-home population, John et al. (2014) did find a clear association in a cohort of Swiss patients receiving home-care services; and though this was a frail population more similar to our institutionalised subjects, $20 \%$ were nevertheless aged under 65 years.

With specific regard to institutionalised populations, Donaldson and Jagger (1983) observed a higher mortality associated with frequent urinary incontinence in a sample of old persons in hospitals and homes for older people, but with limited confounding control. Again with insufficient adjustment, Lewis et al. (1985) reported a higher short-term risk in a sample of discharged residents from 24 nursing homes, as did Ekelund and Rundgren (1987), without any adjustment, in long-term care patients.

To sum up, a critical view of a possible causal role for incontinence shows that only two studies with suitably adjusted analyses found a positive association (Thom et al. 1997, John et al. 2014) and both of these were conducted on community-dwelling populations. A third community-based study found some evidence of an association (Nuotio et al. 2002).

Although a few well-designed studies have observed a positive association in essentially community-dwelling populations (Thom et al. 1997, Nuotio et al. 2002), the failure of many others to ensure control for pre-existing morbidity conditions has meant that potential associations might have been confounded by these factors (Johnson et al. 2000, Holroyd-Leduc 
et al. 2004, Berardelli et al. 2013) including those in institutional populations. Additionally, whatever the results in a community-dwelling population might be, it might not be possible to extrapolate the results to an institutionalised population, since the two differ in many respects.

The role of urinary incontinence as a mortality marker, i.e. a factor associated with conditions which, in turn, increase the risk of dying, has not been called into question. However, the plausibility of its causal implication understandably enjoys less credibility. Since testing this hypothesis through experimental designs is far from easy, non-experimental studies with a sufficient set of measured confounding factors may provide an appropriate alternative design for the purpose.

\title{
THE STUDY
}

\begin{abstract}
Aims
The main objective of this study was to measure the potential causal association between baseline urinary incontinence and long-term, all-cause mortality in a representative sample of institutionalised older adults, using a wide range of relevant baseline covariates to control for confounding and explore effect modification.

\section{Design}

We conducted a cohort study, using mortality follow-up data drawn from a baseline survey of a probabilistic sample of residents of residential and nursing homes aged 65 years or over, in the city of Madrid (Spain) during the period June 1998-June 1999.
\end{abstract}

\section{Participants}


We selected a baseline probabilistic sample of residents, aged 65 years and over, of public and private nursing homes in the city of Madrid (Spain)and a surrounding area of up to $35 \mathrm{~km}$ distant. Study participants were selected through stratified cluster sampling, including one stratum with 22 public and 25 subsidized (privately owned but publicly funded) nursing homes and another stratum with 139 private institutions. As a first stage, we sampled 25 public/subsidized and 30 private institutions with probability proportional to their sizes. As a second stage the interviewers obtained a list of all the residents from the director of each facility and then they selected 10 men and 10 women in each public/subsidized facility chosen and five men and five women from each private nursing home chosen by means of a systematic sampling with random start (with the aid of random number tables). Four private institutions (totalling 40 sample subjects) refused participation and 45 additional residents could not be selected due to absence or refusal, leading to an overall response rate of $89 \%$ ( 715 of the 800 sample residents). Due to refusal, prolonged absence or sampling frame errors, thirty-nine subjects were randomly replaced with residents of the same facility and sex, with the consequence that information could be gathered through structured interviews with 754 residents.

\section{Data-collection}

Using structured questionnaires, baseline data were collected by geriatricians or residents in geriatrics - who had been given appropriate interviewing instruction - through interviews conducted with participants, their main caregivers and facility physicians.

Medical conditions, including cancer, chronic obstructive pulmonary disease (COPD), arrhythmias, hypertension, ischaemic heart disease, congestive heart failure, peripheral arterial disease, stroke, diabetes, anaemia, dementia, Parkinson's disease, epilepsy, depression, anxiety disorders and arthritis, were ascertained by interviewing nursing-home physicians (or nurses for 
$8 \%$ of residents) with access to patients' medical histories. Details of medications used in the preceding 7 days were obtained by reviewing medical records. The total number of medical conditions and prescribed medications was then computed.

Functional status was evaluated by means of the modified Barthel Index (Shah et al. 1989). Fifty-nine percent of subjects were asked about their degree of dependence in performing basic activities of daily living (ADL). The same information on the remaining $41 \%$ of residents was obtained from their main caregivers. The Barthel Index ADLs are: eating; going to the toilet; personal hygiene; bathing/showering; dressing/undressing; transferring; walking; use of stairs; and urinary/faecal continence. For each activity there are five response levels, which yield an overall score ranging from 0 (totally dependent) to 100 points (functionally independent). For the purposes of our study, however, we opted for an ad hoc version of the index which excluded the continence items and had an overall score range of 0 to 80 points. Residents were then classified as being functionally independent (80 points), mildly to moderately dependent (31-79 points) or severely to totally dependent (0-30 points).

In the case of urinary incontinence, study subjects or their caregivers were asked whether the resident had experienced any involuntary leakage of urine in the preceding 14 days (as used by in the Minimum Data Set (Morris et al. 1990)). Response options were: 1. No; 2. Occasionally (1-2 episodes); 3. Only at night (with diurnal control); 4. Frequent (3-6 episodes); or 5. Total (several episodes, during the day and at night). Urinary incontinence was defined as any involuntary leakage of urine in the preceding 14 days (options 2 to 5), response options 2 or 3 (occasional or only nocturnal) were classified as mild incontinence and 4 or 5 (frequent or total) as severe incontinence.

\section{Mortality during follow-up}


Mortality was ascertained by mailing a survey to the participating facilities requesting updated data on residents' vital status and by linkage to the National Death Index (Ministerio de Sanidad 2014). Residents were followed from baseline interview to death, age 105 years or 15 September 2013, whichever occurred first.

\section{Ethical considerations}

The "Carlos III” Institute of Health Institutional Review Board approved the study. Oral informed consent, duly documented, was obtained from all study participants or their next of kin.

\section{Data-analysis}

The association between urinary incontinence and all-cause mortality was analysed using Cox proportional hazards models, with age as the time-scale to control for age in all models. To adjust for other potential confounders, we used three models with increasing levels of adjustment. The first basic model was adjusted for sex, type of facility (public, subsidized or private) and facility size (number of beds). The second intermediate model was further adjusted for number of medical conditions (continuous), number of prescribed medications (continuous) and functional dependency (independent, mild/moderate or severe/total). The third comprehensive model replaced the number of medical conditions in the second model with indicators for the following specific diseases: cancer, COPD, arrhythmias, hypertension, congestive heart failure, stroke, diabetes, anaemia and dementia. Further adjustment for ischaemic heart disease in $84 \%$ of residents with available data yielded similar results (data not shown). To explore potential effect modification, we included interaction terms between urinary incontinence (yes or no) and each dichotomised covariate in the fully-adjusted Cox proportional hazards model. The proportion of the mortality rate attributable to urinary incontinence was estimated by using Miettinen's formula for the population attributable fraction (Miettinen 1974) which comprises the fully-adjusted 
hazard ratio and the weighted prevalence of urinary incontinence among deaths. The corresponding $95 \%$ confidence interval was computed by means of the variance formula proposed by Greenland (1999). Non-parametric adjusted survival curves for residents with no, mild or severe urinary incontinence were estimated as the baseline survival functions from a Cox model stratified by incontinence group and adjusted to the overall weighted means of all baseline confounders. To allow for non-proportional hazards over time (age), we fitted an age-dependent Cox model with interactions between mild and severe incontinence indicators and a restricted quadratic spline function of age with knots at 80, 90 and 100 years (Hess 1994). Proportional hazards were contrasted by using joint Wald tests for spline interaction coefficients.

Due to the sampling design and the different selection probabilities of study participants (residents in public/subsidized facilities and men were oversampled), all statistical analyses were weighted and took into account the effect of stratification and clustering on point and interval estimates. All reported $P$ values were two-sided. Analyses were performed using the Stata 13.1 (Stata Corp., College Station, Texas) and R 2.15 (R Foundation for Statistical Computing, Vienna, Austria) statistical software programmes.

\section{Reliability and validity}

We used a probabilistic sampling to enhance external validity. Geriatricians and residents in geriatrics - properly trained as interviewers - contributed to a high response rate and to reliable data collection. Most information was collected using validated and known instruments like the Barthel Index and key questions of the Minimum Data Set. Reliability of medical information, including prevalence of diseases and medications, was enhanced by interviewing the facility physicians (nurses in $8 \%$ of the cases) with the aid of the corresponding medical records. Baseline data were checked for errors and inconsistencies in the database and doubtful 
information provided on follow up was discarded. Potential for confounding was reduced through the use of multivariate models including a set of numerous relevant variables.

\section{RESULTS}

Of the 754 participants in the baseline survey, 55 with unknown vital status on termination of follow-up and 24 without valid information on their baseline urinary incontinence status were excluded, thus leaving a final cohort of 675 residents. The median length of stay at baseline was 3.2 years. The residents' previous living arrangements were: with family (52.3\%), alone (31.4\%), other care home $(15.0 \%)$ and hostel or related $(1.3 \%)$. The level of care was considered low if no caregiver was assigned (58.7\%).

A total of 576 participants died across the 4061 person-years of follow-up (median/maximum follow-up: 4.6/15.2 years). Mortality rates were lower among women and residents of intermediate-sized private facilities and increased steadily with age, number of medical conditions and prescribed medications, degree of functional dependency and urinary incontinence (Table 1). Table 2 shows the residents' baseline characteristics by urinary incontinence status, with notable associations in most variables.

In the first basic model, adjusted for age, sex and facility characteristics, the hazard ratio for all-cause mortality for residents with urinary incontinence, as opposed to those without, was 1.55 (95\% confidence interval [CI]: 1.31-1.82) (Table 3). After adjustment for number of medications, functional dependency and a comprehensive list of specific chronic conditions, the association attenuated $(\mathrm{HR}=1.24)$. There was a graded relationship, with hazard ratios of 1.07 and 1.44 for mild and severe urinary incontinence respectively $(P$ for trend $=0.005)($ Table 3$)$. This gradient was also evident in the adjusted survival curves (Figure 1). The proportion of the mortality rate attributable to urinary incontinence was 10.5\% (95\% CI: $1.1-19.0 \%)$. 
The potential for an age-dependent effect of urinary incontinence on mortality was similarly explored. The hazard ratio for mild incontinence varied little at ages below 90 years $(P$ for proportional hazards $=0.27)$, unlike that for severe incontinence $(P$ for proportional hazards $=$ .001 ), which displayed more marked effects at younger ages (hazard ratios of 3.38, 2.64, 2.06 and 1.63 at 75, 80, 85 and 90 years of age respectively) (Figure 2). The association between mild and severe incontinence and mortality was diluted or even reversed at very advanced ages, mainly due to the differential depletion of residents with urinary incontinence.

In subgroup analyses, risk of death associated with urinary incontinence tended to be higher among men, residents with multiple prescribed medications and those with some degree of functional dependency (Figure 3). With regard to specific chronic conditions, residents suffering from COPD, arrhythmias, heart failure and anaemia displayed a higher risk of urinaryincontinence-related mortality, though subgroup-specific estimates were imprecise for less prevalent conditions.

\section{DISCUSSION}

We found a clear, consistent, graded association between urinary incontinence and mortality in an institutionalised population. This association persisted after successive adjustments for relevant variables. Furthermore, it was consistent across several subgroups, including those that were apparently healthier.

In accordance with others' views and our study results, we believe that urinary incontinence is associated with mortality as part of a complex web of frailty and geriatric syndromes (Tinetti et al. 1995, Inouye et al. 2007, Dubeau et al. 2010) making it very difficult to 
isolate its specific role. This is the reason why we think that adjustment for a comprehensive set of relevant variables that form part of the causal pathway between frailty and mortality would have led to a notable weakening in the effect estimates. Even so, our clear finding of an association between urinary incontinence and mortality suggests that, apart from its involvement in frailty processes, urinary incontinence may have a more direct causal role. This implies that a successful intervention (meaningful improvement in patient status) might be followed by a longer survival time. Some mediators in this causal pathway are plausible: for instance, the literature is consistent in showing an association between urinary incontinence and falls (Close 2001, Chiarelli et al. 2009) and between falls and mortality in older people (Nurmi et al. 2004). Galizia et al. (2012) reported an association between falls and long-term mortality, though only in subjects with nocturia. Urinary incontinence can also limit patients' mobility, resulting in a less active lifestyle and affecting social and personal relationships - with long-term consequences in terms of depression, disability and death. Other potential pathways between urinary incontinence and mortality to be considered include urinary tract infections (Mody and Juthani-Mehta 2014) and skin problems (rashes, skin infections and sores) (Kunin et al. 1992, Ersser et al. 2005). It should be stressed here that, when it comes to frail older adults, apparently innocuous factors can trigger a chain of consequences with a fatal outcome.

Faecal incontinence has a close association with urinary incontinence and part of the observed effect could be due to this association. In trying to explore this aspect we added faecal incontinence to the fully adjusted model and found a small reduction (hazard ratios of 1.07 [0.80$1.42]$ and 1.36 [1.02-1.80]) for the mild and severe levels respectively, as compared with no incontinence). Akpan et al. (2007) found faecal incontinence to be associated with 3-month 
mortality but, as recognised by these authors, such effect may be more related to the underlying illness than the incontinence itself.

\section{Strengths and limitations}

Positive aspects that should be highlighted are the use of a representative sample with a very high response rate and the participation of various types of residents, features which enhance our study's external validity. The appreciable sample size of the study limits random error and the availability of a large number of relevant variables confers special importance on a study, when in studies of this type confounding control is seen as paramount. On the other hand, our results are also consistent with other causal pathways, such as frailty, falls, impaired mobility, urinary infections and skin problems, which have been shown to increase mortality. Some limitations may have influenced the results, e.g. between half and two-thirds of residents were interviewed directly, while others' information was collected through caregivers; those who could not be interviewed might conceivably have been in a worse state of health and thus could have had a higher mortality risk, while those who were interviewed may have displayed some bias in their responses, presumably towards underreporting. However, no differences were found, either in the analysis adjusted for important health-related variables or in the search for potential heterogeneity. Cognitive status, an important variable, was not included in the models, due to the high number of missing values; instead, dementia status was used as a surrogate, which should, for the most part, have controlled any confounding effect from the non-inclusion of cognitive status data. As for the outcome, we believe that some deaths might not have been reported, something that would eventually generate non-differential misclassification and, in general, lead to underestimation of the effects. Losses to follow up were scarce and not associated with incontinence status $(P=0.65)$. Lastly, though the set of adjustment variables was comprehensive, 
some degree of residual confounding is still possible. It should, however, be borne in mind here that adjusting for numerous relevant and well-measured covariates, as we did, could improve control of confounding, since these variables may collectively serve as proxies for unmeasured factors (Schneeweiss et al. 2009).

\section{Implications for practice and research}

Our findings bring to light the crucial role of nursing in managing urine incontinence in nursing homes. However, there is room for improving education and training on urinary incontinence for nurses and nursing assistants in nursing homes (Saxer et al. 2008, Mandl et al. 2015). Although there is some evidence showing the positive role of a nurse specialist in the cost-effectiveness of treatment of urinary incontinence in primary care (Holtzer-Goor et al. 2015), benefits from an extension of such professional input to nursing-home settings are not yet established. The hypothesis that urinary incontinence has a causal role for mortality in nursing homes should be tested in future randomised controlled trials, preferably cluster trials. In these trials, interventions to manage urinary incontinence should be compared with current approaches using mortality and health-related quality of life as outcome variables. Potential interventions to be studied may include, among others: education and training for nurses, increasing the role of nurse specialists and integrated continence care in nursing homes (Wagg et al. 2008). Recently, Roe et al. (2015) al carried out a systematic review and found some conservative interventions to be effective in the management of urinary incontinence, though in the short term. These have to be considered in future intervention trials to evaluate a possible effect on the risk of mortality in nursing homes. These trials will have to rely heavily on the leading role of nursing in both their design and conduct.

\section{Conclusions}


Our results lend reasonable support to the idea that urinary incontinence is not only a marker, but also a real determinant of survival for institutionalised older adults. Accordingly, effective interventions might well lead to life-expectancy gains, in addition to other benefits in terms of quality of life. While further investigation is thus needed to ascertain whether the observed association is indeed causal, parallel intervention studies aimed at testing this hypothesis should nevertheless be carried out. The role of nursing in improving the management of urine incontinence in nursing homes - through education and training, a greater role for nurse specialists or integrated continence care - should be studied. 


\section{Acknowledgments}

Ana Villaverde Hueso, pharmacist at the Rare Diseases Research Institute ("Carlos III" Institute of Health), undertook the identification and classification of medications.

We gratefully acknowledge the contribution of all residents and staff of the participating facilities.

\section{Funding}

This work was supported by grant PI15CIII/00037 from the "Carlos III" Institute of Health..

\section{Conflict of interest}

The authors declare that they have no conflicts of interest with respect to this study. 


\section{REFERENCES}

Abrams, P., Andersson, K.E., Birder, L., Brubaker, L., Cardozo, L., Chapple, C., Cottenden, A., Davila, W., de Ridder, D., Dmochowski, R., Drake, M., Dubeau, C., Fry, C., Hanno, P., Smith, J.H., Herschorn, S., Hosker, G., Kelleher, C., Koelbl, H., Khoury, S., Madoff, R., Milsom, I., Moore, K., Newman, D., Nitti, V., Norton, C., Nygaard, I., Payne, C., Smith, A., Staskin, D., Tekgul, S., Thuroff, J., Tubaro, A., Vodusek, D., Wein, A., Wyndaele, J.J., Members of, C. \& Fourth International Consultation on, I. (2010) Fourth International Consultation on Incontinence Recommendations of the International Scientific Committee: Evaluation and treatment of urinary incontinence, pelvic organ prolapse, and fecal incontinence. Neurourology and Urodynamics 29(1), 213-240.

Akpan, A., Gosney, M.A. \& Barret, J. (2007) Factors contributing to fecal incontinence in older people and outcome of routine management in home, hospital and nursing home settings. Clinical Interventions in Aging 2(1), 139-145.

Berardelli, M., De, R.F., Morelli, M., Corsonello, A., Mazzei, B., Mari, V., Montesanto, A., Lattanzio, F. \& Passarino, G. (2013) Urinary incontinence in the elderly and in the oldest old: correlation with frailty and mortality. Rejuvenation Research 16(3), 206-211.

Close, J.C. (2001) Interdisciplinary practice in the prevention of falls--a review of working models of care. Age and Ageing 30 Suppl 4, 8-12.

Chiarelli, P.E., Mackenzie, L.A. \& Osmotherly, P.G. (2009) Urinary incontinence is associated with an increase in falls: a systematic review. Australian Journal of Physiotherapy 55(2), 89-95. 
Donaldson, L.J. \& Jagger, C. (1983) Survival and functional capacity: three year follow up of an elderly population in hospitals and homes. Journal of Epidemiology and Community Health 37(3), 176-179.

Dubeau, C.E., Kuchel, G.A., Johnson, T., Palmer, M.H. \& Wagg, A. (2010) Incontinence in the frail elderly: report from the 4th International Consultation on Incontinence. Neurourology and Urodynamics 29(1), 165-178.

Ekelund, P. \& Rundgren, A. (1987) Urinary incontinence in the elderly with implications for hospital care consumption and social disability. Archives of Gerontology and Geriatrics 6(1), 11-18.

Ersser, S.J., Getliffe, K., Voegeli, D. \& Regan, S. (2005) A critical review of the interrelationship between skin vulnerability and urinary incontinence and related nursing intervention. International Journal of Nursing Studies 42(7), 823-35.

Galizia, G., Langellotto, A., Cacciatore, F., Mazzella, F., Testa, G., Della-Morte, D., Gargiulo, G., Ungar, A., Ferrara, N., Rengo, F. \& Abete, P. (2012) Association between nocturia and falls-related long-term mortality risk in the elderly. Journal of the American Medical Directors Association 13(7), 640-4.

Greenland, S. (1999) Re: "Confidence limits made easy: interval estimation using a substitution method" [letter]. American Journal of Epidemiology 149(9), 884.

Herzog, A.R., Diokno, A.C., Brown, M.B., Fultz, N.H. \& Goldstein, N.E. (1994) Urinary incontinence as a risk factor for mortality. Journal of the American Geriatrics Society 42(3), 264-268. 
Hess, K.R. (1994) Assessing time-by-covariate interactions in proportional hazards regression models using cubic spline functions. Statistics in Medicine 13(10), 1045-1062.

Holroyd-Leduc, J.M., Mehta, K.M. \& Covinsky, K.E. (2004) Urinary incontinence and its association with death, nursing home admission, and functional decline. Journal of the American Geriatrics Society 52(5), 712-718.

Holtzer-Goor, K.M., Gaultney, J.G., van Houten, P., Wagg, A.S., Huygens, S.A., Nielen, M.M., Albers-Heitner, C.P., Redekop, W.K., Rutten-van Molken, M.P. \& Al, M.J. (2015) CostEffectiveness of Including a Nurse Specialist in the Treatment of Urinary Incontinence in Primary Care in the Netherlands. PloS One 10(10), e0138225.

Inouye, S.K., Studenski, S., Tinetti, M.E. \& Kuchel, G.A. (2007) Geriatric syndromes: clinical, research, and policy implications of a core geriatric concept. Journal of the American Geriatrics Society 55(5), 780-791.

John, G., Gerstel, E., Jung, M., Dallenbach, P., Faltin, D., Petoud, V., Zumwald, C. \& Rutschmann, O.T. (2014) Urinary incontinence as a marker of higher mortality in patients receiving home care services. British Journal of Urology International 113(1), 113-119.

Johnson, T.M., Bernard, S.L., Kincade, J.E. \& DeFriese, G.H. (2000) Urinary incontinence and risk of death among community-living elderly people: results from the National Survey on Self-Care and Aging. Journal of Aging and Health 12(1), 25-46.

Jones, A.L., Dwyer, L.L., Bercovitz, A.R. \& Strahan, G.W. (2009) The National Nursing Home Survey: 2004 overview. Vital and Health Statistics. Series 13: Data from the National Health Survey (167), 1-155. 
Kojima, G. (2015) Prevalence of Frailty in Nursing Homes: A Systematic Review and MetaAnalysis. Journal of the American Medical Directors Association 16(11), 940-5.

Kunin, C.M., Douthitt, S., Dancing, J., Anderson, J. \& Moeschberger, M. (1992) The association between the use of urinary catheters and morbidity and mortality among elderly patients in nursing homes. American Journal of Epidemiology 135(3), 291-301.

Lewis, M.A., Kane, R.L., Cretin, S. \& Clark, V. (1985) The immediate and subsequent outcomes of nursing home care. American Journal of Public Health 75(7), 758-762.

Mandl, M., Halfens, R.J. \& Lohrmann, C. (2015) Incontinence care in nursing homes: a cross-sectional study. Journal of Advanced Nursing 71(9), 2142-52.

Miettinen, O.S. (1974) Proportion of disease caused or prevented by a given exposure, trait or intervention. American Journal of Epidemiology 99(5), 325-32.

Milsom, I., Coyne, K.S., Nicholson, S., Kvasz, M., Chen, C.I. \& Wein, A.J. (2014) Global prevalence and economic burden of urgency urinary incontinence: a systematic review. European Urology 65(1), 79-95.

Ministerio de Sanidad, S.S.e.I. (2014) Spanish National Death Index. . Vol. 2014.

Mody, L. \& Juthani-Mehta, M. (2014) Urinary tract infections in older women: a clinical review. JAMA 311(8), 844-54.

Morris, J.N., Hawes, C., Fries, B.E., Philips, C.D., Mor, V., Katz, S., Murphy, K., Drugovich, M.L. \& Friedlob, A.S. (1990) Designing the national resident assessment instrument for nursing homes. Gerontologist 30(3), 293-307. 
Nuotio, M., Tammela, T.L., Luukkaala, T. \& Jylha, M. (2002) Urgency and urge incontinence in an older population: ten-year changes and their association with mortality. Aging Clinical and Experimental Research 14(5), 412-419.

Nurmi, I.S., Luthje, P.M. \& Kataja, J.M. (2004) Long-term survival after falls among the elderly in institutional care. Archives of Gerontology and Geriatrics 38(1), 1-10.

Offermans, M.P., Du Moulin, M.F., Hamers, J.P., Dassen, T. \& Halfens, R.J. (2009)

Prevalence of urinary incontinence and associated risk factors in nursing home residents: a systematic review. Neurourology and Urodynamics 28(4), 288-294.

Roe, B., Flanagan, L. \& Maden, M. (2015) Systematic review of systematic reviews for the management of urinary incontinence and promotion of continence using conservative behavioural approaches in older people in care homes. Journal of Advanced Nursing 71(7), 1464-83.

Saxer, S., de Bie, R.A., Dassen, T. \& Halfens, R.J. (2008) Nurses' knowledge and practice about urinary incontinence in nursing home care. Nurse Education Today 28(8), 926-34.

Schneeweiss, S., Rassen, J.A., Glynn, R.J., Avorn, J., Mogun, H. \& Brookhart, M.A. (2009) High-dimensional propensity score adjustment in studies of treatment effects using health care claims data. Epidemiology 20(4), 512-522.

Shah, S., Vanclay, F. \& Cooper, B. (1989) Improving the sensitivity of the Barthel index for stroke rehabilitation. Journal of Clinical Epidemiology 42(8), 703-709. 
Thom, D.H., Haan, M.N. \& van den Eeden, S.K. (1997) Medically recognized urinary incontinence and risks of hospitalization, nursing home admission and mortality. Age and Ageing 26(5), 367-374.

Tilvis, R.S., Hakala, S.M., Valvanne, J. \& Erkinjuntti, T. (1995) Urinary incontinence as a predictor of death and institutionalization in a general aged population. Archives of Gerontology and Geriatrics 21(3), 307-315.

Tinetti, M.E., Inouye, S.K., Gill, T.M. \& Doucette, J.T. (1995) Shared risk factors for falls, incontinence, and functional dependence. Unifying the approach to geriatric syndromes. JAMA 273, 1348-1353.

Wagg, A., Potter, J., Peel, P., Irwin, P., Lowe, D. \& Pearson, M. (2008) National audit of continence care for older people: management of urinary incontinence. Age and Ageing 37(1), 39-44. 


\section{Figure legends}

Figure 1. Adjusted survival curves from age 75 years for nursing-home residents with no, mild and severe urinary incontinence in the city of Madrid (Spain): 1998-1999 to 2013. Nonparametric survival curves were obtained as the baseline survival functions from a Cox model, stratified by urinary incontinence group (no, mild or severe) and adjusted to the overall weighted means of sex, type of facility (public, subsidized or private), number of beds, number of prescribed medications, functional dependency (independent, mild/moderate or severe/total), cancer, chronic obstructive pulmonary disease, arrhythmias, hypertension, congestive heart failure, stroke, diabetes, anaemia and dementia (Alzheimer's disease or other dementias). Figure 2. Age-dependent hazard ratios for all-cause mortality for nursing-home residents with no, mild and severe urinary incontinence compared with those without incontinence in the city of Madrid (Spain): 1998-1999 to 2013.

Curves represent adjusted hazard ratios (solid lines) and their 95\% confidence intervals (dashed lines) obtained from an age-dependent Cox model with interactions between mild and severe incontinence indicators and a restricted quadratic spline function of age with knots at 80,90 and 100 years, adjusted for sex, type of facility (public, subsidized or private), number of beds, number of prescribed medications, functional dependency (independent, mild/moderate or severe/total), cancer, chronic obstructive pulmonary disease, arrhythmias, hypertension, congestive heart failure, stroke, diabetes, anaemia and dementia (Alzheimer's disease or other dementias). The histograms represent the weighted numbers of person-years by age interval among residents with no incontinence (shaded bars), mild incontinence (top white bars) and severe incontinence (bottom white bars). 
Figure 3. Hazard ratios for all-cause mortality associated with urinary incontinence in subgroups of nursing-home residents in the city of Madrid (Spain): 1998-1999 to 2013.

Subgroup-specific hazard ratios (squares with area inversely proportional to the variance) and their 95\% confidence intervals (CIs, horizontal lines) were obtained from Cox models with interaction terms between urinary incontinence (yes or no) and the corresponding subgroup indicator, adjusted for sex, type of facility (public, subsidized or private), number of beds, number of prescribed medications, functional dependency (independent, mild/moderate or severe/total), cancer, chronic obstructive pulmonary disease, arrhythmias, hypertension, congestive heart failure, stroke, diabetes, anaemia and dementia (Alzheimer's disease or other dementias). 
Table 1. Population Distribution and All-Cause Mortality Rates by Age Interval and Baseline Characteristics of Residents in Nursing Homes in the City of Madrid (Spain), 1998-1999 to 2013.

\begin{tabular}{|c|c|c|c|c|}
\hline & $\begin{array}{c}\text { No. of } \\
\text { subjects }^{\mathrm{a}}(\%)\end{array}$ & $\begin{array}{l}\text { No. of } \\
\text { deaths }\end{array}$ & $\begin{array}{c}\text { No. of } \\
\text { person-years }\end{array}$ & $\begin{array}{c}\text { Mortality rate } \\
(95 \% \text { CI })\end{array}$ \\
\hline Overall & $675(100)$ & 576 & 4061 & $137.0(124.3-150.8)$ \\
\hline \multicolumn{5}{|l|}{ Age interval (years) } \\
\hline $65-74$ & $94(13.0)$ & 32 & 290 & $109.3(77.2-157.2)$ \\
\hline $75-84$ & $337(47.7)$ & 132 & 1327 & $93.7(78.1-112.8)$ \\
\hline $85-94$ & $474(72.2)$ & 309 & 1905 & $157.5(138.8-178.7)$ \\
\hline $95-104$ & $154(24.3)$ & 103 & 539 & $174.1(135.1-223.6)$ \\
\hline \multicolumn{5}{|l|}{ Sex } \\
\hline Women & $374(75.9)$ & 319 & 2318 & $133.9(118.9-150.6)$ \\
\hline Men & $301(24.1)$ & 257 & 1743 & $147.8(128.1-170.1)$ \\
\hline \multicolumn{5}{|l|}{ Type of facility } \\
\hline Public & $390(47.1)$ & 346 & 2317 & $148.7(132.6-166.4)$ \\
\hline Subsidized & $66(7.6)$ & 54 & 354 & $167.4(122.1-227.7)$ \\
\hline Private & $219(45.3)$ & 176 & 1390 & $122.0(103.0-144.1)$ \\
\hline \multicolumn{5}{|c|}{ Facility size (no. of beds) } \\
\hline$<100$ & $131(26.6)$ & 113 & 741 & $148.3(120.5-181.6)$ \\
\hline $100-299$ & $263(39.3)$ & 214 & 1640 & $118.9(100.6-140.2)$ \\
\hline$\geq 300$ & $281(34.1)$ & 249 & 1680 & $151.9(132.8-173.4)$ \\
\hline \multicolumn{5}{|c|}{ No. of medical conditions } \\
\hline $0-1$ & $147(21.9)$ & 121 & 1034 & $122.4(100.9-148.2)$ \\
\hline $2-3$ & $280(41.8)$ & 236 & 1818 & $123.0(106.3-142.1)$ \\
\hline$\square 4$ & $248(36.3)$ & 219 & 1209 & $170.2(142.6-202.3)$ \\
\hline \multicolumn{5}{|c|}{ No. of prescribed medications } \\
\hline $0-2$ & $168(26.0)$ & 142 & 1113 & $124.6(103.5-149.7)$ \\
\hline $3-4$ & $225(35.7)$ & 195 & 1330 & $144.8(122.8-170.3)$ \\
\hline$\square 5$ & $252(38.3)$ & 212 & 1440 & $138.7(117.1-163.7)$ \\
\hline \multicolumn{5}{|c|}{ Functional dependency } \\
\hline Independent & $222(28.4)$ & 183 & 1761 & $96.1(83.0-111.0)$ \\
\hline Mild/moderate & $305(49.6)$ & 261 & 1736 & $142.1(123.0-163.8)$ \\
\hline Severe/total & $133(22.0)$ & 118 & 488 & $227.6(172.8-296.5)$ \\
\hline
\end{tabular}


Urinary incontinence

\begin{tabular}{llrrl} 
No & $334(46.5)$ & 278 & 2435 & $111.1(98.4-125.3)$ \\
Yes & $341(53.5)$ & 298 & 1626 & $170.5(146.0-198.4)$ \\
$\quad$ Mild & $121(18.7)$ & 101 & 719 & $127.7(99.8-162.7)$ \\
$\quad$ Severe & $220(34.8)$ & 197 & 907 & $203.6(166.7-247.1)$ \\
\hline
\end{tabular}

a Unweighted sample counts and weighted percentages. Figures across age intervals add up to more than the overall sample size because subjects may contribute to different age intervals during follow-up.

${ }^{b}$ Weighted mortality rates per 1,000 person-years and 95\% confidence intervals (CIs). 
Table 2. Baseline Characteristics of the Residents in Nursing Homes in the City of Madrid (Spain), 1998-1999, with and without Urinary Incontinence.

\begin{tabular}{|c|c|c|c|}
\hline & Urinar & ence a & \\
\hline Variable & No & Yes & $P$-value $\mathrm{b}$ \\
\hline Age (mean, y) & 82.2 & 84.2 & 0.004 \\
\hline Sex (\% women) & 45.3 & 54.7 & 0.22 \\
\hline Type of facility & & & $<0.001$ \\
\hline Public & 57.9 & 42.1 & \\
\hline Subsidized & 25.7 & 74.3 & \\
\hline Private & 38.1 & 61.9 & \\
\hline Facility size (no. of beds) & & & $<0.001$ \\
\hline$<100$ & 33.7 & 66.3 & \\
\hline $100-299$ & 44.0 & 56.0 & \\
\hline$\geq 300$ & 59.4 & 40.6 & \\
\hline No. of medical conditions & & & $<0.001$ \\
\hline $0-1$ & 61.6 & 38.4 & \\
\hline $2-3$ & 48.2 & 51.8 & \\
\hline$\square 4$ & 35.4 & 64.6 & \\
\hline No. of prescribed medications & & & 0.11 \\
\hline $0-2$ & 53.2 & 46.8 & \\
\hline $3-4$ & 47.4 & 52.6 & \\
\hline$\square 5$ & 39.7 & 60.3 & \\
\hline Dementia & 19.5 & 80.6 & $<0.001$ \\
\hline Functional dependency & & & $<0.001$ \\
\hline Independent & 93.4 & 6.6 & \\
\hline Mild/moderate & 50.0 & 50.0 & \\
\hline Severe/total & 3.6 & 96.5 & \\
\hline
\end{tabular}

a Weighted percentages.

b Two-sided tests 
Table 3. Hazard Ratios for All-Cause Mortality Associated with Baseline Urinary Incontinence and Its Severity Levels in Residents of Nursing Homes in the City of Madrid (Spain), 1998-1999 to 2013 .

\begin{tabular}{|c|c|c|c|}
\hline \multirow[b]{2}{*}{ Urinary incontinence } & \multicolumn{3}{|c|}{ Hazard ratio ${ }^{a}(95 \%$ CI $)$} \\
\hline & Model 1b & Model 2c & Model 3d \\
\hline No & 1.00 (reference) & 1.00 (reference) & 1.00 (reference) \\
\hline Yes & $1.55(1.31-1.82)$ & $1.27(1.04-1.56)$ & $1.24(1.02-1.50)$ \\
\hline Mild & $1.14(0.88-1.48)$ & $1.08(0.81-1.46)$ & $1.07(0.80-1.41)$ \\
\hline Severe & $1.89(1.57-2.28)$ & $1.52(1.18-1.95)$ & $1.44(1.12-1.85)$ \\
\hline$P$-value for trend $\mathrm{e}$ & $<0.001$ & 0.002 & 0.005 \\
\hline
\end{tabular}

${ }^{a}$ Hazard ratios and 95\% confidence intervals (CIs) were obtained from Cox proportional hazards models with age as time scale and three increasing levels of adjustment.

${ }^{\mathrm{b}}$ Model 1 adjusted for age, sex, type of facility (public, subsidized, or private), and number of beds.

- Model 2 additionally adjusted for number of medical conditions, number of prescribed medications, and functional dependency (independent, mild/moderate, or severe/total).

${ }^{a}$ Model 3 replaced the number of medical conditions in Model 2 with indicators for the following specific diseases: cancer, chronic obstructive pulmonary disease, arrhythmias, hypertension, congestive heart failure, stroke, diabetes, anaemia, and dementia (Alzheimer's disease or other dementias).

e $P$-value for log-linear trend in hazard ratios across urinary incontinence severity levels (no, mild, and severe). 


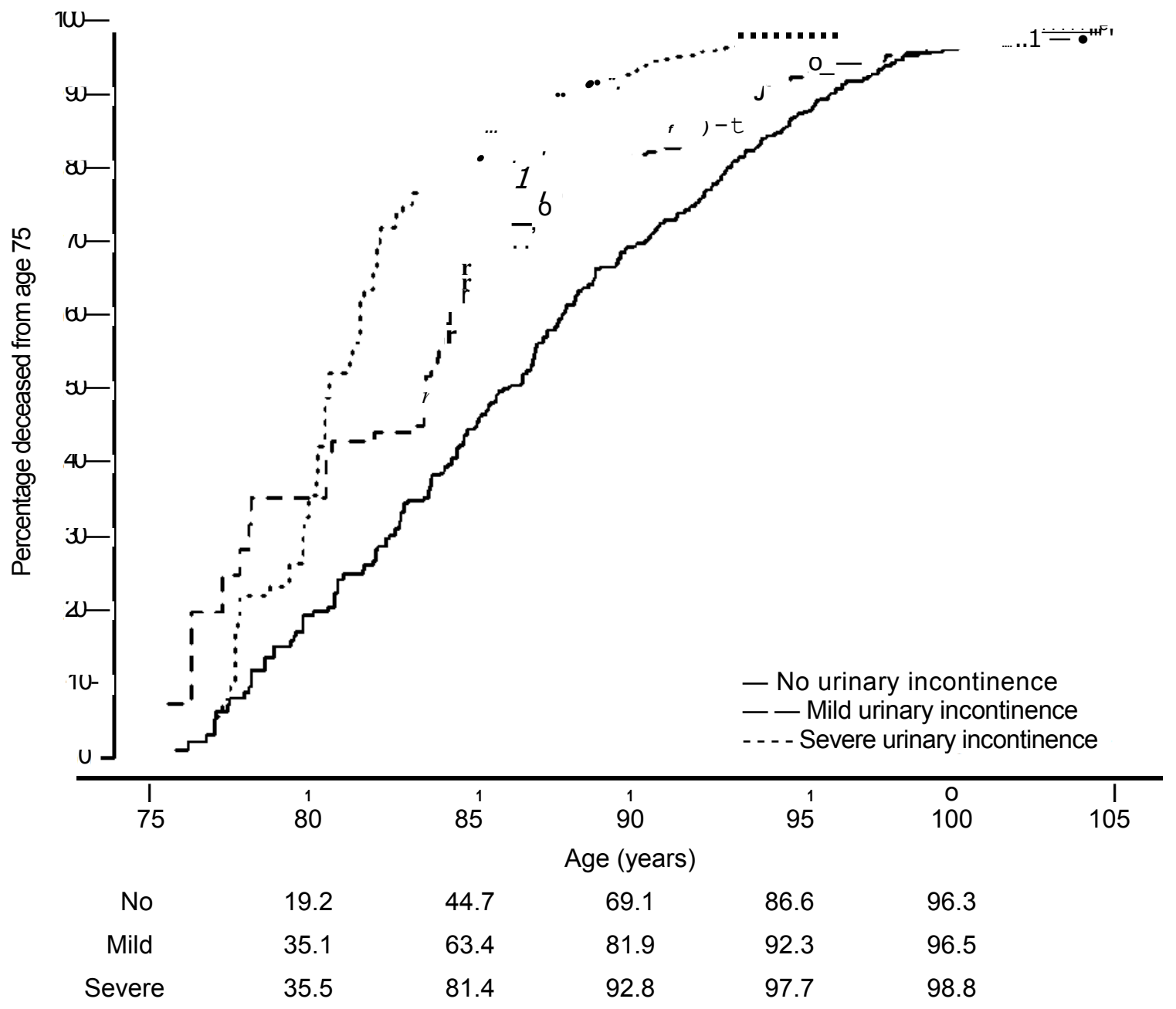



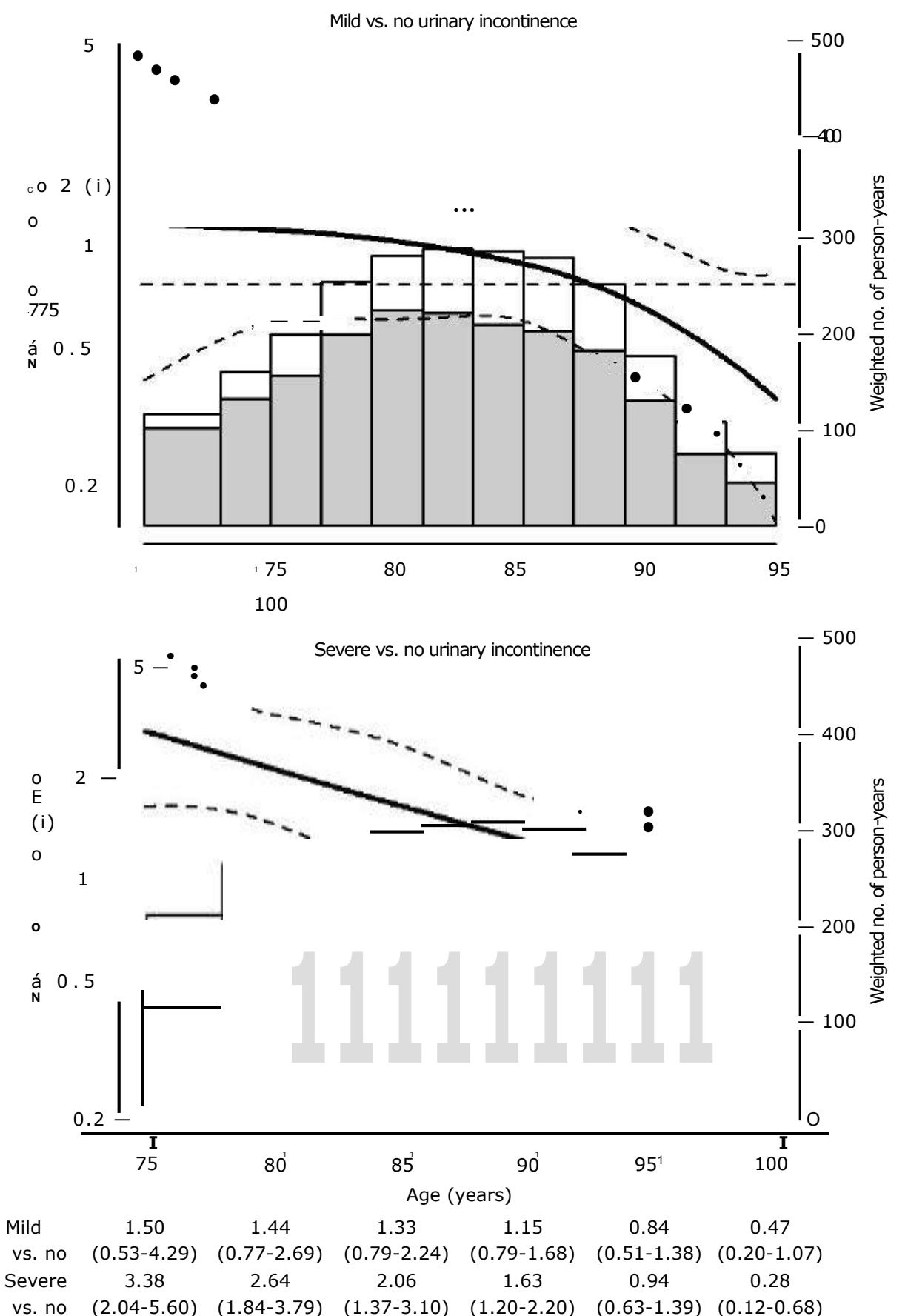


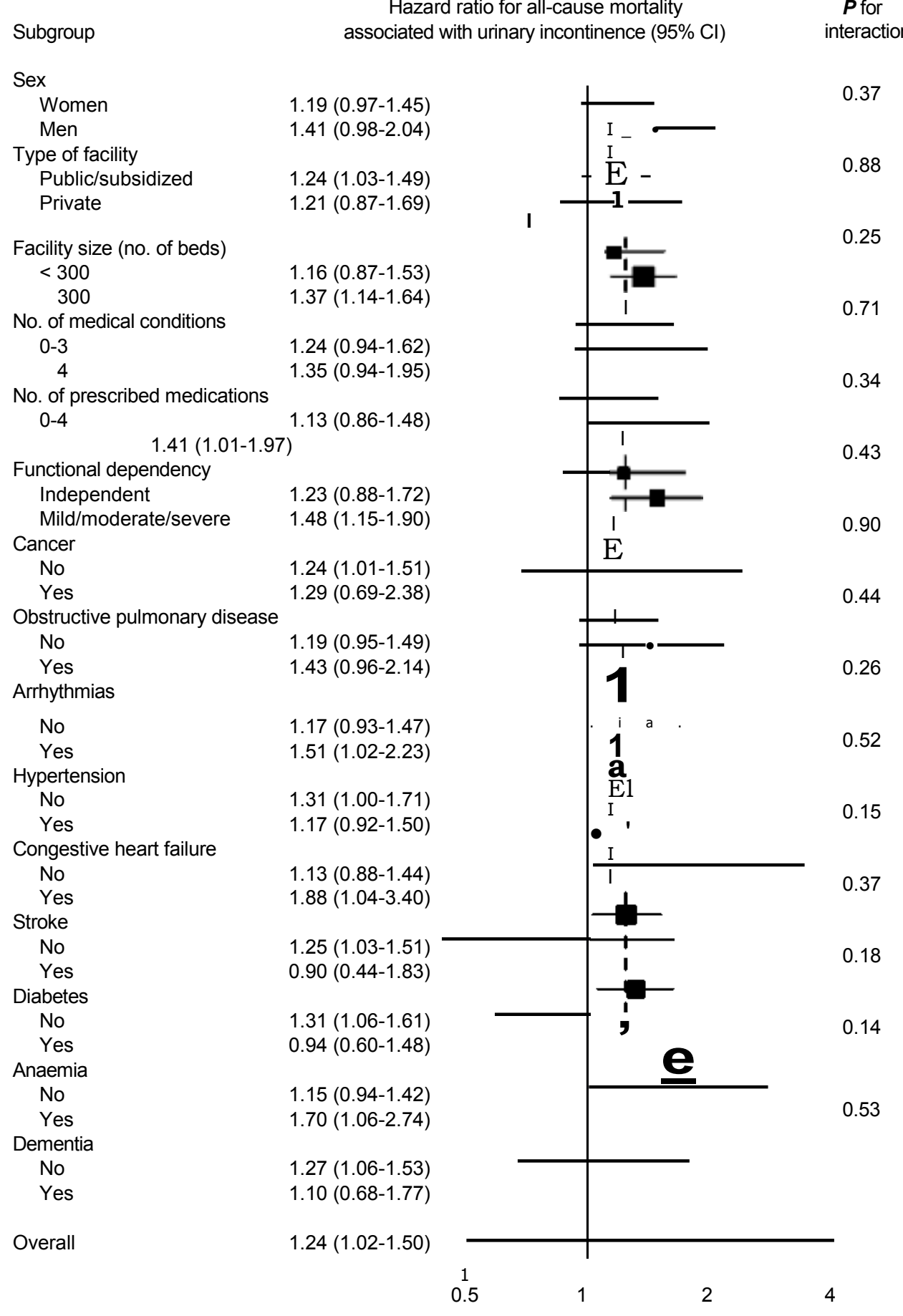

